\title{
Chemical analysis by ultrahigh-resolution nuclear magnetic resonance in the Earth's magnetic field
}

\author{
STEPHAN APPELT1* ${ }^{*}$ HOLGER KÜHN ${ }^{1}$, F. WOLFGANG HÄSING ${ }^{1}$ AND BERNHARD BLÜMICH² \\ ${ }^{1}$ Zentralinstitut für Elektronik, Forschungszentrum Jülich, D-52425 Jülich, Germany \\ ${ }^{2}$ Institut für Technische Chemie und Makromolekulare Chemie, RWTH Aachen, D-52056 Aachen, Germany \\ *e-mail: st.appelt@fz-juelich.de
}

H igh-resolution NMR spectroscopy is a powerful tool for non-destructive structural investigations of matter ${ }^{1}$. Typically, expensive and immobile superconducting magnets are required for chemical analysis by high-resolution NMR spectroscopy. Here we present the feasibility of liquid-state proton $\left({ }^{1} \mathrm{H}\right)$, lithium $\left({ }^{7} \mathrm{Li}\right)$ and fluorine $\left({ }^{19} \mathrm{~F}\right)$ ultrahigh-resolution NMR spectroscopy ${ }^{2}$ in the Earth's magnetic field. We show that in the Earth's field the transverse relaxation time $T_{2}$ of the ${ }^{7} \mathrm{Li}$ nucleus is very sensitive to its mobility in solution. The $J$-coupling constants ${ }^{3}$ of silicon-containing $\left({ }^{29} \mathrm{Si}\right)$ and fluorine-containing molecules are measured with just a single scan. The accuracy of the measured ${ }^{1} \mathrm{H}-{ }^{29} \mathrm{Si}$ and ${ }^{1} \mathrm{H}-{ }^{19} \mathrm{~F}$ $J$-coupling constants is between a few millihertz up to $20 \mathrm{mHz}$. This is at least one order of magnitude better than the precision obtained with superconducting magnets. The high precision allows the discrimination of similar chemical structures of small molecules as well as of macromolecules.

Increasing requirements of sensitivity and of spectral dispersion have driven the development of NMR magnets to higher and more homogeneous magnetic fields, which are obtained by immobile and expensive superconducting magnets. With the best field homogeneities available $\left(\Delta B / B \sim 10^{-9}\right.$ over $1 \mathrm{~cm}^{3}$, where $B$ is the magnetic field), ultrahigh-resolution carbon $\left({ }^{13} \mathrm{C}\right)$ NMR spectra at $4.2 \mathrm{~T}$ with an instrumental broadening below $50 \mathrm{mHz}$ have been realized ${ }^{2}$. For ${ }^{1} \mathrm{H}$ high-field NMR spectroscopy $(1-20 \mathrm{~T})$, it is difficult to measure linewidths with an instrumental broadening below $100 \mathrm{mHz}$.

We define our ideal NMR spectrometer by two requirements: first, it should measure NMR spectra with high resolution and all relevant NMR parameters, such as the longitudinal $\left(T_{1}\right)$ and transverse $\left(T_{2}\right)$ relaxation times, the chemical shift and the dipolar and $J$-coupling, in a single scan; and second, the spectrometer should be robust, low cost and mobile. Low cost and mobile means that heavy electro or superconducting magnets as well as superconducting quantum interference devices (SQUIDs) should be avoided. Single-scan and high-resolution NMR implies

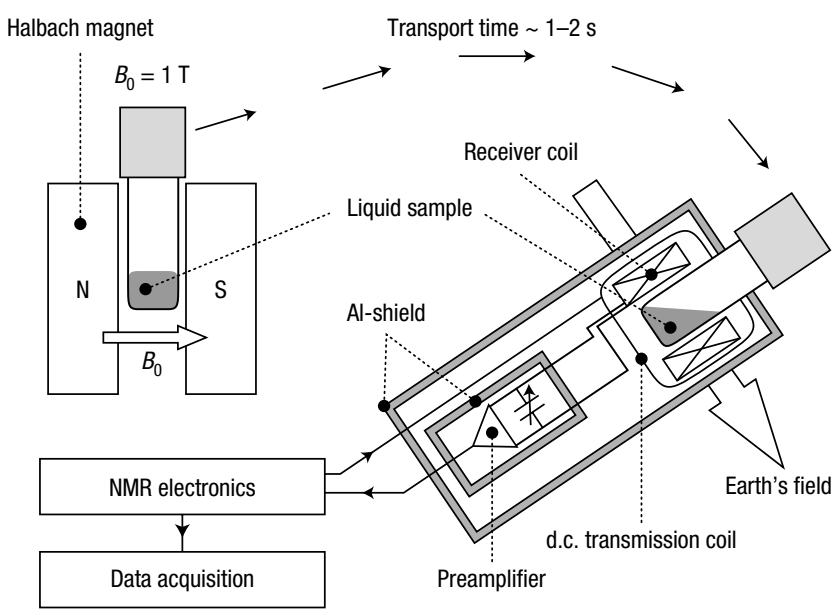

Figure 1 Setup of mobile ultrahigh-resolution Earth's field NMR. The nuclear spins of a liquid sample are first prepolarized by using a Halbach magnet and then transported to the NMR probe of the spectrometer. After a $90^{\circ}$ d.c. pulse excitation, the FID of the nuclear spins in the Earth's magnetic field $\left(\sim 5 \times 10^{-5} \mathrm{~T}\right)$ is acquired and processed.

that sufficiently high premagnetization of the spins and a very homogeneous magnetic field are required. Do we come closer to this ideal by performing NMR in low field or in the Earth's magnetic field $\left(2.5-7.5 \times 10^{-5} \mathrm{~T}\right)$ ? Important steps towards mobile chemical-shift-resolved low-field NMR in inhomogeneous fields have been shown ${ }^{4,5}$. The first NMR spectra in the Earth's field were demonstrated by Packard and Varian ${ }^{6}$. NMR has been performed in the Earth's field in order to measure the ${ }^{1} \mathrm{H}-{ }^{31} \mathrm{P}$ and ${ }^{1} \mathrm{H}-{ }^{14} \mathrm{~N} J$-coupling constants ${ }^{7,8}$. Self-diffusion and relaxation-time measurements ${ }^{9-11}$, proton magnetic resonance imaging ${ }^{11}$ (MRI), 

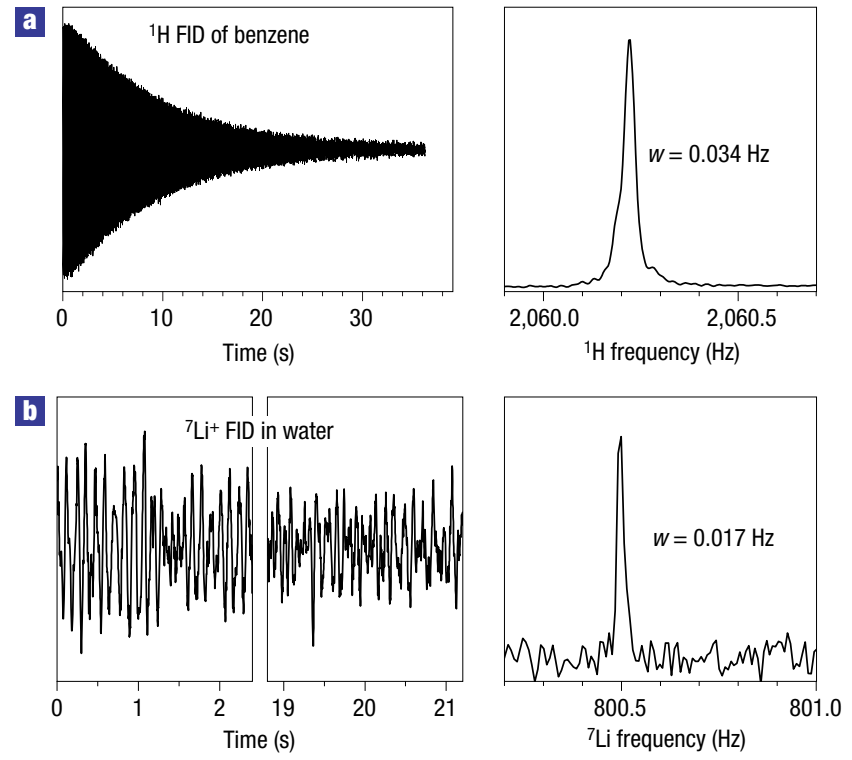

$\mathbf{G}$

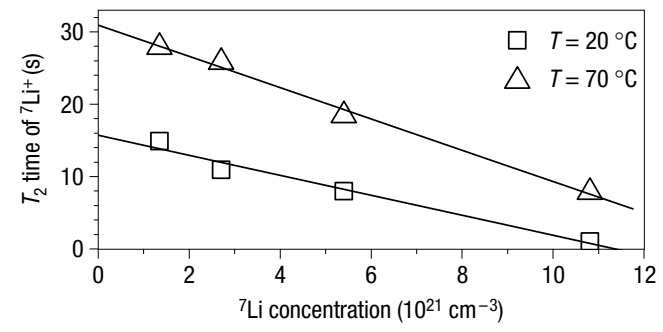

Figure 2 Single-scan ${ }^{1} \mathrm{H}$ and ${ }^{7} \mathrm{Li}$ NMR signals measured in the Earth's field. a, ${ }^{1} \mathrm{H}$ FID of $2 \mathrm{~cm}^{3}$ deoxygenated benzene (left) and the corresponding Fourier-transformed spectrum (right). The linewidth $w$ is $0.034 \mathrm{~Hz}$ full-width at half-maximum. Note the absolute scale of the frequency axis of the spectrum, showing a ${ }^{1} \mathrm{H}$-Larmor frequency of 2,060.22020 $\pm 3 \times 10^{-5} \mathrm{~Hz}$. b. Single-scan FID of the ${ }^{7} \mathrm{Li}+$ ion in solution at $T=70^{\circ} \mathrm{C}$ and the corresponding spectrum (right). The ${ }^{7} \mathrm{Li}$-Larmor frequency is $800.4967 \pm 4 \times 10^{-4} \mathrm{~Hz}$ and the linewidth is

$0.017 \pm 2 \times 10^{-3} \mathrm{~Hz}$ full-width at half-maximum. c, $T_{2}$-relaxation time of ${ }^{7} \mathrm{Li}^{+}$ions in water as a function of the ${ }^{7} \mathrm{Li}^{+}$concentration. The squares (triangles) correspond to a sample temperature of $20^{\circ} \mathrm{C}\left(70^{\circ} \mathrm{C}\right)$. The solid lines are guides for the eye.

the detection of groundwater reservoirs ${ }^{12}\left(>1 \mathrm{~m}^{3}\right)$ and the determination of water diffusion in the pores of the Antarctic shelf ice ${ }^{13}$ were achieved by Earth's field NMR. Heteronuclear $J$-coupling constants have been measured with a spectral resolution of about $1 \mathrm{~Hz}$ in the micro- and nanotesla regime $e^{14-16}$ by using SQUID sensors ${ }^{17,18}$.

Two facts are important for the application of NMR in the Earth's field. First, for most spin species, the chemical shift cannot be resolved. This is owing to the fact that the spectral lines are normally much broader than the line separation induced by chemical-shift differences. One exception is hyperpolarized xenon $\left({ }^{129} \mathrm{Xe}\right)$, which allows Earth's field $\mathrm{Xe}$ chemical-shift measurements in liquids with a precision comparable to that of superconducting magnets ${ }^{19}$. Second, only heteronuclear J-couplings can be measured in the Earth's field, because the chemical-shift differences for a given nuclear-spin species $(<0.1 \mathrm{~Hz})$ are smaller than the typical values of the homonuclear $J$-coupling constants $(\sim 0.5-200 \mathrm{~Hz})$.

In this contribution, we report single-scan measurements of heteronuclear J-coupled resolved NMR spectra in the Earth's magnetic field with an accuracy of a few millihertz. Very narrow lines are observed for ${ }^{1} \mathrm{H},{ }^{7} \mathrm{Li}$ and ${ }^{19} \mathrm{~F}$ nuclei in the liquid state. The origin of these narrow lines is that far enough away $(>100 \mathrm{~m})$ from buildings and steel constructions the Earth's field is very homogeneous $\left(\triangle B / B<10^{-6}\right)$ over a sample volume of $1 \mathrm{~cm}^{3}$. For nuclei with $T_{1} \sim T_{2}>3 \mathrm{~s}$, the linewidth is well below $0.1 \mathrm{~Hz}$, and the instrumental broadening of Earth's field NMR is smaller than a few millihertz. In the case of thermally polarized samples with a volume of a few $\mathrm{cm}^{3}$, the signal-to-noise ratio $(\mathrm{S} / \mathrm{N})$ is smaller than $10^{-2}$ for coil-based Earth's field NMR ${ }^{20}$. Therefore, the nuclear spins have to be premagnetized to a value of at least 10,000 times the Boltzmann magnetization at $5 \times 10^{-5} \mathrm{~T}$. Advanced hyperpolarization methods $\mathrm{s}^{21-27}$ and the use of mobile electromagnets ${ }^{13}$ are successful premagnetization techniques, but the most simple mobile and robust tool is a cylindrical Halbach magnet ${ }^{28}$ with a typical field strength of $1-2 \mathrm{~T}$ inside the bore and a negligible field outside (see Fig. 1). The following liquid samples with a volume of $2 \mathrm{~cm}^{3}$ were investigated in the Earth's field: benzene $\left(\mathrm{C}_{6} \mathrm{H}_{6}\right)$, lithium chloride $\left({ }^{7} \mathrm{LiCl}\right)$ dissolved in water, tetramethylsilane (TMS), silicone oil (Baysilone M10), octamethylcyclotetrasiloxane (CTS) and nonafluorohexene (NFH).

Figure 2a, b shows two examples of experimental singlescan free induction decays (FIDs). Long $T_{2}$-relaxation times are observed for the ${ }^{1} \mathrm{H}$ signal of deoxygenated benzene $\left(T_{2}=9.4 \pm\right.$ $0.1 \mathrm{~s}$, see Fig. 2a) and for the ${ }^{7} \mathrm{Li}^{+}$quadrupolar nuclei dissolved in deoxygenated water $\left(T_{2}=18.7 \pm 0.9 \mathrm{~s}\right.$, see Fig. $\left.2 \mathrm{~b}\right)$. The signal-tonoise ratio for one single scan is 100 for the ${ }^{1} \mathrm{H}$ spectrum and about 3 for the ${ }^{7} \mathrm{Li}$ spectrum. A lorentzian fit of the ${ }^{1} \mathrm{H}$ spectrum allows the determination of the ${ }^{1} \mathrm{H}$-Larmor frequency in the Earth's field with an accuracy of $3 \times 10^{-5} \mathrm{~Hz}$. This corresponds to an accuracy of $1 \mathrm{pT}$ for the absolute value of the local Earth's magnetic field. This might be useful for geophysical applications ${ }^{29}$. In Fig. 2b, the concentration of the ${ }^{7} \mathrm{Li}^{+}$ions in water was $5.4 \times 10^{21} \mathrm{~cm}^{-3}\left({ }^{7} \mathrm{Li}\right.$ : nuclear spin $I=3 / 2$, natural abundance $92.6 \%$ ). We found that the ${ }^{7} \mathrm{Li}$ linewidth and the $T_{2}$-relaxation time are extremely sensitive to the temperature and to the ${ }^{7} \mathrm{Li}^{+}$ion concentration. Figure $2 \mathrm{c}$ shows that the ${ }^{7} \mathrm{Li} T_{2}$-relaxation time decreases linearly with the ${ }^{7} \mathrm{Li}^{+}$ion concentration and increases with increasing temperature of the solvent. A maximum value of $T_{2}=31 \mathrm{~s}$ is observed for $\mathrm{T}=70{ }^{\circ} \mathrm{C}$ and for low ${ }^{7} \mathrm{Li}^{+}$concentration.

The results of Fig. 2c can be explained as follows. The $T_{2}$-relaxation time of the ${ }^{7} \mathrm{Li}^{+}$ion in water is dominated by the quadrupolar and by the ${ }^{1} \mathrm{H}-{ }^{7} \mathrm{Li}$ dipole-dipole relaxation mechanisms ${ }^{30}$. In the short-correlation limit $\left(2 \pi v_{\mathrm{Li}} \tau_{\mathrm{c}} \ll 1\right.$, where $v_{\mathrm{Li}}$ is the ${ }^{7} \mathrm{Li}$ Larmor frequency and $\tau_{\mathrm{c}}$ is the correlation time), both relaxation rates depend linearly on $\tau_{c}$, which grows with increasing viscosity of the $\mathrm{LiCl}$ solution. The viscosity is higher at low temperatures and at high ${ }^{7} \mathrm{Li}^{+}$concentration. Note that, for liquids in the Earth's field, the short-correlation limit is valid because $\tau_{c} \ll 10^{-6} \mathrm{~s}$ even for a saturated and viscous $\mathrm{LiCl}$ solution. Owing to the large dynamic range of the $T_{2}$-relaxation time in low field, NMR and MRI measurements of ${ }^{7} \mathrm{Li}^{+}$ions may be important for medical applications and for materials science. For example, MRI could be used for brain-function mapping owing to the change of the ${ }^{7} \mathrm{Li}^{+} T_{2}$-relaxation time in the ion channels of the neurons.

The narrow ${ }^{1} \mathrm{H}$ lines observed in the Earth's field allow accurate measurement of the heteronuclear $J$-coupling constants of molecules. The $J$-coupling constant is a measure of the strength of the electron-mediated indirect nuclear spin-spin coupling ${ }^{3}$. To compare the resolution of high-field NMR with Earth's field NMR, Fig. 3a shows a ${ }^{1} \mathrm{H}-{ }^{29} \mathrm{Si} J$-coupled ${ }^{1} \mathrm{H}$ spectrum of TMS, which has been measured with a well-shimmed superconducting high-field magnet at $9.4 \mathrm{~T}$. Although using a good shimming of $\sim 3$ p.p.b., the ${ }^{1} \mathrm{H}-{ }^{29} \mathrm{Si} J$-coupled doublet with a coupling constant of $J=6.6 \pm 0.1 \mathrm{~Hz}$ can be barely seen in Fig. 3a. The dominant line 

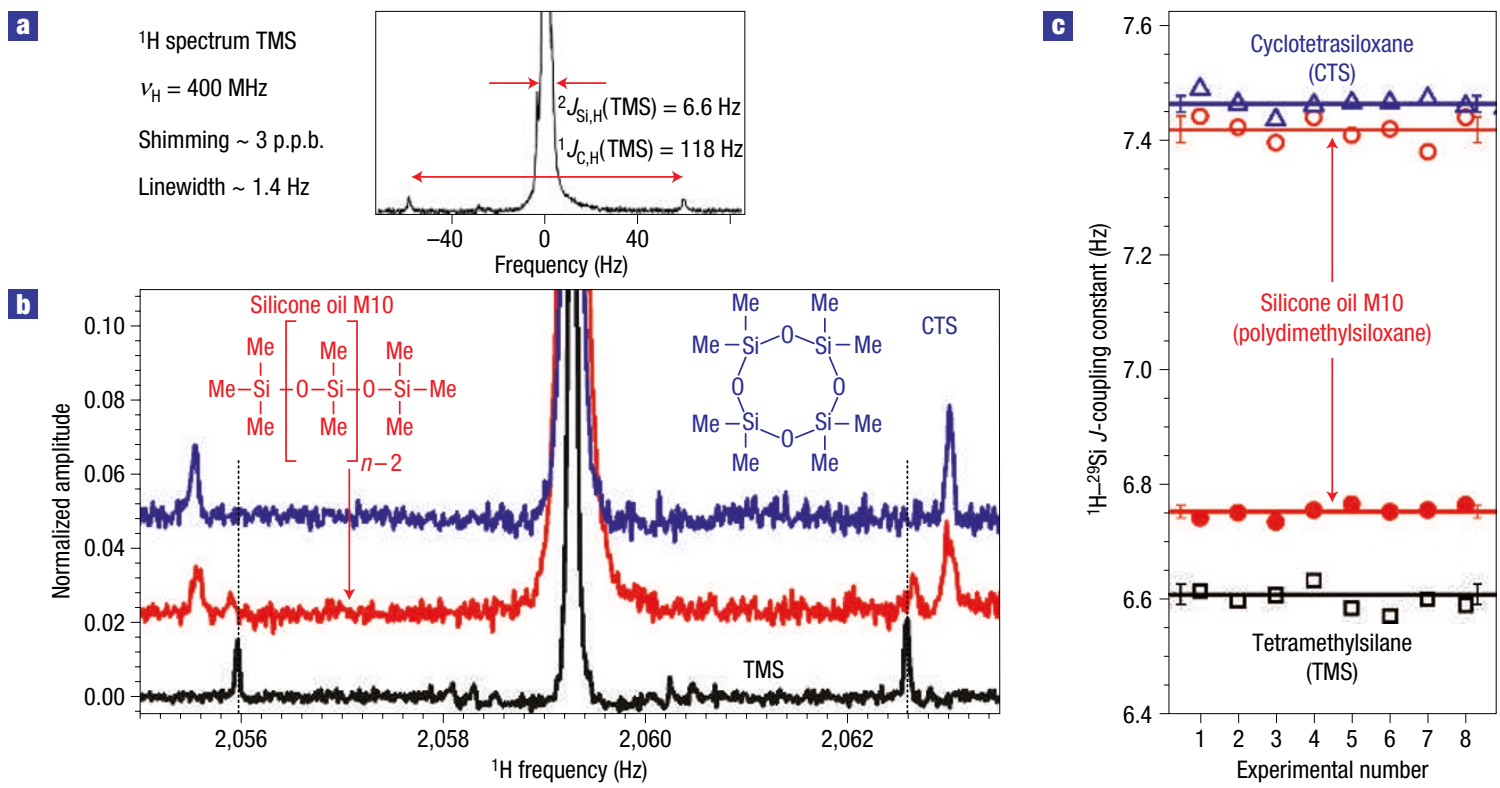

Figure 3 Precision measurements of ${ }^{1} \mathrm{H}-{ }^{29} \mathrm{Si}$ J-couplings for different silicon compounds. a, ${ }^{1} \mathrm{H}$-reference spectrum of TMS measured at the ${ }^{1} \mathrm{H}$ Larmor frequency $v_{H}=400 \mathrm{MHz}$ with a superconducting magnet shimmed to a few p.p.b. (with kind permission of R. Hoffman of the Department of Organic Chemistry, The Hebrew University of Jerusalem). The original p.p.m. scale was rescaled to the frequency scale. b. Single-scan ${ }^{1} \mathrm{H}$ Earth's field NMR spectra of TMS (black), silicone oil M10 (red) and CTS (blue). All three spectra show different values of the ${ }^{1} \mathrm{H}-{ }^{29} \mathrm{Si}$ J-coupling constants. The amplitude of the central peak (which corresponds to $95.3 \%$ of the protons without a $\mathrm{J}$-coupling) is normalized to 1. c, Statistics over eight scans of all measured ${ }^{1} \mathrm{H}-{ }^{29} \mathrm{Si} J$-coupling constants. The squares correspond to TMS, the circles to silicone oil and the triangles to CTS. The fluctuations mainly arise from the imperfect phasing of the spectrum. The error bars indicate the standard deviation of the mean $J$-value (solid lines).

of the protons (width $\sim 1 \mathrm{~Hz}$ ) bound to ${ }^{28} \mathrm{Si}$ and to ${ }^{30} \mathrm{Si}(I=0$, natural abundance $95.3 \%$ ) masks the small doublet of the protons coupled to the ${ }^{29} \mathrm{Si}(I=1 / 2$, natural abundance $4.7 \%)$. Figure $3 \mathrm{~b}$ (black spectrum) shows a proton spectrum of TMS, but measured with a single scan in the Earth's magnetic field. Compared with Fig. 3a, the TMS spectrum is better resolved by a factor of twenty, and the ${ }^{1} \mathrm{H}-{ }^{29} \mathrm{Si} J$-coupling constant of $6.620 \pm 0.002 \mathrm{~Hz}$ can be determined with high accuracy.

The $J$-coupling is very sensitive to the structure and type of the chemical bonds. For example, the ${ }^{1} \mathrm{H}-{ }^{29} \mathrm{Si} J$-coupling depends on the number of oxygen atoms bound to the ${ }^{29} \mathrm{Si}$ atom. This is demonstrated in Fig. $3 \mathrm{~b}$ (blue spectrum), where a single-scan ${ }^{1} \mathrm{H}$ spectrum of CTS is displayed. The chemical structure of the CTS ring is shown on the upper right of Fig. 3b. One single ${ }^{1} \mathrm{H}-{ }^{29} \mathrm{Si}$ $J$-coupling constant with $J=7.470 \pm 0.005 \mathrm{~Hz}$ is observed. This value is significantly different from the $J$-coupling constant of TMS $(6.620 \mathrm{~Hz})$, because in the CTS ring two oxygens are bound to the ${ }^{29} \mathrm{Si}$, which modify the energy of the molecular orbitals of the $\mathrm{SiMe}_{2}$ group (where Me refers to the methyl group). This CTS ring is an important educt in the ring-opening polymerization to long-chained silicone oil molecules (polydimethylsiloxane).

The ${ }^{1} \mathrm{H}-{ }^{29} \mathrm{Si} J$-coupling constants can also be measured for long macromolecular chains. In Fig. $3 b$ (red spectrum) the Earth's field ${ }^{1} \mathrm{H}$ spectrum of silicone oil (Baysilone M10) is shown, which consists of macromolecules with an average chain length of $n=16$, with $14-\left[\mathrm{O}-\mathrm{SiMe}_{2}\right]-$ repeat units, and two terminal $-\mathrm{O}-\mathrm{SiMe}_{3}$ groups (see Fig. $3 \mathrm{~b}$ upper left). The spectrum points out two distinct ${ }^{1} \mathrm{H}-{ }^{29} \mathrm{Si} J$-coupling constants, one with $J=7.420 \pm 0.005 \mathrm{~Hz}$ corresponding to the $-\left[\mathrm{O}-\mathrm{SiMe}_{2}\right]-$ group and another with $J=6.78 \pm 0.01 \mathrm{~Hz}$ corresponding to the terminal $-\mathrm{O}-\mathrm{SiMe}_{3}$ groups. The peak integral below one $J$-coupled line is proportional to the total number of methyl groups. In our case, the ratio of the peak integrals between the large and the small $J$-coupled lines is about 2.7. Obviously, the silicone oil has more -O-SiMe ${ }_{3}$ groups than expected. For a linear molecular chain with an average total chain length of $n=16$, the expected peak-integral ratio would be $28 / 6=4.66$. This means that the silicone oil consists of branched molecules, and there are more than two $-\mathrm{O}-\mathrm{SiMe}_{3}$ groups per molecule. An overview of all measured ${ }^{1} \mathrm{H}-{ }^{29} \mathrm{Si} J$-coupling constants for TMS, silicone oil and CTS is given in Fig. 3c. For each molecule, eight single-scan measurements have been performed in order to estimate the statistical errors of the measured $J$-coupling constants. Note that the two $J$-coupling constants $J=7.47 \mathrm{~Hz}$ for CTS and $J=7.42 \mathrm{~Hz}$ for the silicone oil belong to the same chemical group $-\left[\mathrm{O}-\mathrm{SiMe}_{2}\right]-$, but they differ by $0.05 \mathrm{~Hz}$. The $\mathrm{O}-\mathrm{Si}-\mathrm{O}$ bonding angle of the CTS ring must be slightly distorted and thus it differs from that in the silicone oil. The ability to discriminate between the molecular structure of the CTS ring and the silicone oil opens the possibility for the mobile online characterization of a polymerization reaction.

The splitting due to the ${ }^{1} \mathrm{H}-{ }^{19} \mathrm{~F} J$-coupling in trifluoroethanol has been detected at $\sim 1 \mu \mathrm{T}$ using SQUID NMR ${ }^{17,18}$, and the linewidths of the ${ }^{1} \mathrm{H}$ and ${ }^{19} \mathrm{~F}$ resonances were about $1 \mathrm{~Hz}$. Figure 4 shows a complicated case, the ${ }^{19} \mathrm{~F}$ spectrum of NFH. In the Earth's field, the NFH molecule is characterized by eight heteronuclear ${ }^{1} \mathrm{H}-{ }^{19} \mathrm{~F} J$-coupling constants. Homonuclear ${ }^{19} \mathrm{~F}-{ }^{19} \mathrm{~F} J$-couplings are not observable. Therefore, the spectrum is a superposition of four triplets and four doublets. In the weak-coupling limit $\left(J \ll\left|v_{\mathrm{H}}-v_{\mathrm{F}}\right|=121.7 \mathrm{~Hz}\right.$, where $v_{\mathrm{H}}$ and $v_{\mathrm{F}}$ are the ${ }^{1} \mathrm{H}$ and ${ }^{19} \mathrm{~F}$ Larmor frequencies, respectively), each observed triplet and doublet can be assigned to a $J$-coupling constant $J_{\mathrm{F}-\mathrm{Ha}}$ and $J_{\mathrm{F}-\mathrm{Hb}}$, respectively. The largest value is ${ }^{3} J_{\mathrm{F}-\mathrm{Hb}}=3.58 \mathrm{~Hz}$ (doublet $\mathrm{d} 3$ ), where proton $\mathrm{Hb}$ is coupled over three bonds to the two ${ }^{19} \mathrm{~F}$ spins of a $\mathrm{CF}_{2}$ group. The smallest value is ${ }^{7} J_{\mathrm{F}-\mathrm{Ha}}=0.19 \mathrm{~Hz}$ (triplet $\mathrm{t} 7$ ), where the two protons $\mathrm{Ha}$ are coupled over seven bonds to the three ${ }^{19} \mathrm{~F}$ spins of the $\mathrm{CF}_{3}$ group. The $J$-coupling values over four, five and 


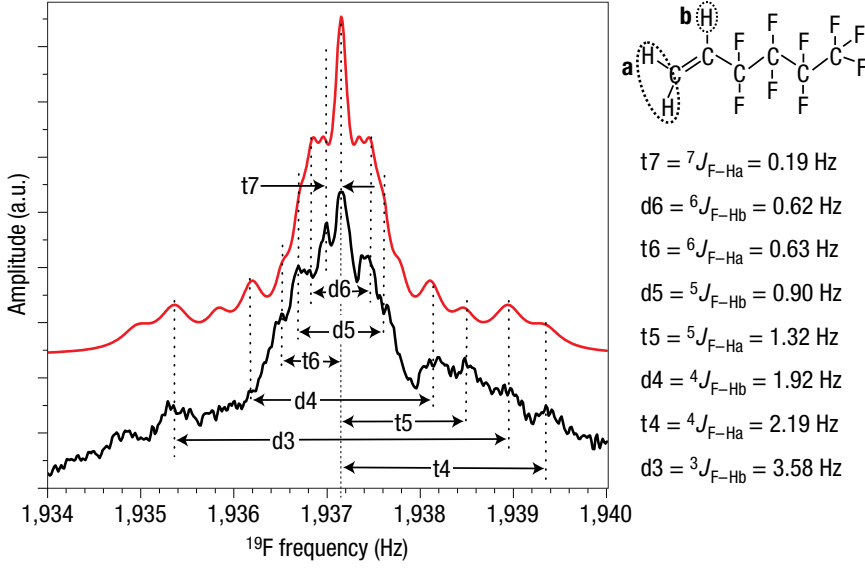

Figure 4 Experimental and simulated ${ }^{19} \mathrm{~F}$ Earth's field NMR spectra of NFH. The NFH structure is shown in the upper right corner. Each proton species, labelled as $\mathrm{Ha}$ and $\mathrm{Hb}$, is $\mathrm{J}$-coupled to the ${ }^{19} \mathrm{~F}$ nuclei of three $\mathrm{CF}_{2}$ groups and one $\mathrm{CF}_{3}$ group. The spectrum is a superposition of four doublets (d3-d6, where, for example, $\mathrm{d} 3 \mathrm{stands}$ for a doublet with three bonds between ${ }^{19} \mathrm{~F}$ and ${ }^{1} \mathrm{Hb}$ ) and four triplets (t4-t7) with eight corresponding ${ }^{1} \mathrm{H}-{ }^{19} \mathrm{~F} J$-coupling constants, as indicated by the arrows. The $J$-coupling constants are plotted on the right. The black (red) line represents the experimental (simulated) NFH spectrum. For the experimental spectrum, nine scans have been averaged in order to improve the signal-to-noise ratio. The parameters for the simulation are the eight $J$-coupling constants, and the linewidths $(0.16-0.40 \mathrm{~Hz})$ are extracted from the NFH spectrum. The integrated intensity of each multiplet spectrum is chosen to be proportional to the number of fluorine atoms of the corresponding chemical group $\left(\mathrm{CF}_{2}\right.$ and $\left.\mathrm{CF}_{3}\right)$.

six bonds have been assigned by arguing that the coupling constant decreases monotonically with increasing number of bonds, and that a double bond results in a larger $J$-coupling constant than a single bond. The simulation (the red line in Fig. 4) of the ${ }^{19} \mathrm{~F}$ spectrum of NFH obtained by a superposition of four triplets and four doublets reflects the basic features of the measured spectrum.

We have shown that the molecular structure can be explored by single-scan ultrahigh-resolution NMR in the Earth's field. In future, the potential of low-field NMR could be further improved by combining the methods of multidimensional $\mathrm{NMR}^{1}$ with advanced mobile hyperpolarization technologies (spin-exchange optical pumping, SEOP ${ }^{21-24}$; para-hydrogen-induced polarization transfer, $\mathrm{PHIP}^{25,26}$; and spin-polarization-induced nuclear Overhauser effect, SPINOE ${ }^{27}$ ). In low magnetic fields, these techniques will allow time-resolved NMR measurements of rare spins such as ${ }^{6} \mathrm{Li},{ }^{13} \mathrm{C}$ and ${ }^{29} \mathrm{Si}$, and the separation of their corresponding spin interactions with high precision. Many applications of mobile low-field NMR in medicine and materials science are likely. This includes the NMR and MRI of hyperpolarized ${ }^{6} \mathrm{Li}^{+}$and ${ }^{7} \mathrm{Li}^{+}$ migrating through ion channels or membranes, the measurement of biomagnetic fields, the detection of tiny changes of the Earth's field in geologically active regions, the characterization of mineral oil in well logging, and the online detection of chemical reactions.

\section{METHODS}

\section{SAMPLE PREPARATION}

An NMR glass tube ( $20 \mathrm{~mm}$ outer diameter) equipped with a valve was filled with $2 \mathrm{~cm}^{3}$ of the liquid sample. All samples were deoxygenated by several freezing and thawing cycles under high-vacuum conditions. Finally, the samples were flushed with ${ }^{4} \mathrm{He}$ gas at a few bars' pressure, and the valve closed.

\section{NMR SETUP}

The Halbach permanent magnet ${ }^{28}$ with a bore of $50 \mathrm{~mm}$ was composed of eight magnetized FeNd segments, which were combined in a cylinder with an outer diameter of $130 \mathrm{~mm}$ and a height of $150 \mathrm{~mm}$. The field strength in the centre was about $1 \mathrm{~T}$. The weak stray field outside the magnet typically broadens a proton NMR line at $1 \mathrm{~m}$ distance to about $0.1 \mathrm{~Hz}$. This broadening was avoided by increasing the distance between the Halbach magnet and the NMR probe to several metres. The probe had two components: a cylindrical receiver coil (with a few thousand windings of copper wire) and a saddle coil for the spin excitation. The $90^{\circ}$ pulse was produced by the saddle coil in terms of a d.c. magnetic field of strength $B_{1}=1.8 \times 10^{-4} \mathrm{~T}$ for a duration of $50-150 \mu$ s. The direction of the $B_{1}$ field was orthogonal to the direction of the Earth's magnetic field vector. The probe was $60 \mathrm{~cm}$ away from the preamplifier, which had a voltage noise of $<2 \mathrm{nV} \mathrm{Hz}^{1 / 2}$. A 20-mm-thick aluminium cylinder provided a shielding from ambient electromagnetic noise. The signal after preamplification was processed by a home-made lock-in amplifier, which operated at a reference frequency close to the Larmor frequency of the spins. Typically, the detection bandwidth was $20 \mathrm{~Hz}$. The whole receiver electronics had a noise contribution that was smaller than the Johnson noise of the receiver coil $(\sim 10 \mathrm{nV})$. Therefore, our NMR setup was operating close to the Johnson noise limit.

\section{MEASUREMEN}

Nuclear spins with a longitudinal relaxation time $T_{1}>1 \mathrm{~s}$ were premagnetized with a 1 T Halbach magnet (Fig. 1), and then carried within 1-2 s to the coil of the Earth's field NMR spectrometer. There, transverse magnetization was generated by a $90^{\circ}$ d.c. magnetic field pulse with the saddle coil; the subsequent FID was picked up by the NMR receiver coil, amplified and processed.

Received 27 October 2005; accepted 16 December 2005; published 22 January 2006

\section{References}

1. Ernst, R. R., Bodenhausen, G. \& Wokaun, A. Principles of Nuclear Magnetic Resonance in One and Two Dimensions (Clarendon, Oxford, 1987)

2. Allerhand, A., Addleman, R. E. \& Osman, D. Ultrahigh resolution NMR. 1. General considerations and preliminary results for carbon-13 NMR. J. Am. Chem. Soc. 107, 5809-5812 (1985).

3. Proctor, W. G. \& Yu, F. C. On the nuclear magnetic moments of several stable isotopes. Phys. Rev. 81, 20-30 (1951).

4. Meriles, C. A., Sakellariou, D., Heise, H., Moulé, A. J. \& Pines, A. Approach to high resolution ex situ NMR spectroscopy. Science 293, 82-85 (2001).

5. Perlo, J. et al. High-resolution NMR spectroscopy with a portable single-sided sensor. Science $\mathbf{3 0 8}$, 1279 (2005).

6. Packard, M. \& Varian, R. Free nuclear induction in the Earth's magnetic field. Phys. Rev. 93, 941 (1954).

7. Benoit, H., Hennequin, J. \& Ottavi, H. Les applications spectroscopiques de la méthode de prépolarisation en R.M.N. (champs faibles). Chim. Anal. 44, 471-477 (1962).

8. Béné, G. J. Nuclear magnetism of liquid systems in the Earth field range. Phys. Rep. $\mathbf{5 8}$ 213-267 (1980).

9. Stepišnik, J., Kos, M., Planinšič, G. \& Eržen, V. Strong nonuniform magnetic field for self-diffusion measurement by NMR in the Earth's magnetic field. J. Magn. Reson. A 107, 167-172 (1994).

10. Belorizky, E. et al. Translational diffusion constants and intermolecular relaxation in paramagnetic solutions with hyperfine coupling on the electronic site. J. Phys. Chem. A 102, 3674-3680 (1998).

11. Planinsič, G., Stepišnik, J. \& Kos, M. Relaxation-time measurement and imaging in the Earth's magnetic field. J. Magn. Reson. A 110, 170-174 (1994)

12. Shushakov, A. Groundwater NMR in conductive water. Geophysics 61, 998-1006 (1996).

13. Callaghan, P. T., Eccles, C. D. \& Seymour, J. D. An Earth's field nuclear magnetic resonance apparatus suitable for pulsed gradient spin echo measurements of self-diffusion under Antarctic conditions. Rev. Sci. Instrum. 68, 4263-4270 (1997)

14. McDermott, R. et al. Liquid-state NMR and scalar couplings in microtesla magnetic fields. Science 295, 2247-2249 (2002).

15. Trabesinger, A. H. et al. SQUID-detected liquid state NMR in microtesla fields. J. Phys. Chem. A 108, 957-963 (2004).

16. Burghoff, M., Hartwig, S., Trahms, L. \& Bernarding, J. Nuclear magnetic resonance in the nanoTesla range. Appl. Phys. Lett. 87, 054103 (2005).

17. Greenberg, Y. S. Application of superconducting quantum interference devices to nuclear magnetic resonance. Rev. Mod. Phys. 70, 175-222 (1998).

18. Clarke, J. in SQUID Sensors: Fundamentals, Fabrication and Applications (ed. Weinstock, H.) 1-62 (Kluwer Academic, Dordrecht, 1996).

19. Appelt, S., Häsing, F. W., Kühn, H., Perlo, J. \& Blümich, B. Mobile high resolution xenon nuclear magnetic resonance spectroscopy in the Earth's magnetic field. Phys. Rev. Lett. 94, 197602 (2005).

20. Webb, A. G. Radiofrequency microcoils in magnetic resonance. Prog. Nucl. Magn. Reson. Spectrosc 31, 1-42 (1997).

21. Happer, W. Optical pumping. Rev. Mod. Phys. 44, 169-249 (1972).

22. Appelt, S. et al. Theory of spin-exchange optical pumping of ${ }^{3} \mathrm{He}$ and ${ }^{129} \mathrm{Xe}$. Phys. Rev. A 58, 1412-1439 (1998)

23. Bouchiat, M. A., Carver, T. R. \& Varnum, C. M. Nuclear polarization in $\mathrm{He}^{3}$ gas induced by optical pumping and dipolar exchange. Phys. Rev. Lett. 5, 373-375 (1960).

24. Colegrove, F. D., Schearer, L. D. \& Walters, G. K. Polarization of $\mathrm{He}^{3}$ gas by optical pumping. Phys. Rev. 132, 2561-2572 (1963)

25. Bowers, C. R. \& Weitekamp, D. P. Transformation of symmetrization order to nuclear-spin magnetization by chemical reaction and nuclear magnetic resonance. Phys. Rev. Lett. 57, 2645-2648 (1986). 
26. Goldman, M., Jóhannesson, H., Axelsson, O. \& Karlsson, M. Hyperpolarization of ${ }^{13} \mathrm{C}$ through order transfer from parahydrogen: A new contrast agent for MRI. Magn. Reson. Imaging 23, 153-157 (2005)

27. Navon, G. et al. Enhancement of solution NMR and MRI with laser-polarised Xe. Science 271, 1848-1851 (1996).

28. Halbach, K. Design of permanent multipole magnets with oriented rare Earth cobalt material. Nucl. Instrum. Methods 169, 1-10 (1980).

29. Kernevez, N., Duret, D., Moussavi, M. \& Leger, J.-M. Weak field NMR and ESR spectrometers and magnetometers. IEEE Trans. Magn. 28, 3054-3059 (1992).

30. Eliav, U. \& Navon, G. Measurement of dipolar interaction of quadrupolar nuclei in solution using multiple-quantum NMR spectroscopy. J. Magn. Reson. 123, 32-48 (1996).

\section{Acknowledgements}

The authors gratefully acknowledge excellent technical assistance from U. Sieling from the Forschungszentrum Jülich. We are grateful to K. Münnemann and S. Stapf from the ITMC Aachen for the support and the classification of the silicone samples.

Correspondence and requests for materials should be addressed to S.A.

Competing financial interests

The authors declare that they have no competing financial interests.

Reprints and permission information is available online at http://npg.nature.com/reprintsandpermissions/ 